\title{
ON THE SKEWNESS OF ORDER STATISTICS IN MULTIPLE-OUTLIER MODELS
}

\author{
SUBHASH KOCHAR, ${ }^{*}$ Portland State University \\ MAOCHAO XU,** Illinois State University
}

\begin{abstract}
Kochar and $\mathrm{Xu}$ (2009) proved that a parallel system with heterogeneous exponential component lifetimes is more skewed (according to the convex transform order) than the system with independent and identically distributed exponential components. In this paper we extend this study to the general $k$-out-of- $n$ systems for the case when there are only two types of component in the system. An open problem proposed in Păltănea (2008) is partially solved.
\end{abstract}

Keywords: Dispersive order; exponential distribution; decreasing failure rate; $k$-out-of- $n$ system; proportional hazard rate; right spread order; skewness; star order

2010 Mathematics Subject Classification: Primary 60E15; 62N05; 62G30; 62D05

\section{Introduction}

Skewness describes the departure of a distribution from symmetry, where one tail of the density is more 'stretched out' than the other. Several well-known measures of skewness are available in the statistics literature, such as Pearson's coefficient of skewness and Edgeworth's coefficient. Interested readers are referred to Arnold and Groeneveld (1995) and Marshall and Olkin (2007, p. 70) for more discussion and other measures of skewness. Skewed data is observed in many areas, such as economics, engineering, medicine, insurance, and psychology. It may be easy to recognize symmetric distributions, but it is not so easy to determine whether one nonsymmetric distribution is more skewed than another. Several partial orders have been introduced in the literature to compare the relative skewness of probability distributions. Van Zwet (1970) introduced the concept of the convex transform order to compare two distributions according to their skewness. Gamma distributions, which play a prominent role in actuarial science due to their skewness, are ordered according to the convex transform order in terms of their shape parameters. Another well-known partial order to compare the skewness of two probability distributions is the star order (cf. Barlow and Proschan (1981, p. 105) and Oja (1981)). This ordering is weaker than the convex transform order. It is well known that the star order implies the Lorenz order, which is an important partial order in economics to compare income inequalities.

Order statistics have received a great amount of attention in the statistics literature since they are widely used in reliability, data analysis, goodness-of-fit tests, statistical inference, and other applied probability and statistical areas. Most of these studies focused mainly on the case when order statistics are from independent and identically distributed (i.i.d.) random

Received 29 July 2010; revision received 12 October 2010.

* Postal address: Department of Mathematics and Statistics, Portland State University, Portland, Oregon 97201, USA.

** Postal address: Department of Mathematics, Illinois State University, Normal, Illinois 61790-4520, USA.

Email address: mxu2@ilstu.edu 
variables. We refer the reader to David and Nagaraja (2003) and Balakrishnan and Rao (1998a), (1998b) for more details. Studies of order statistics from heterogeneous samples began in the early 1970s, motivated by robustness issues. After that, a lot of work has been done in singleoutlier and multiple-outlier models. Balakrishnan (2007) synthesized recent developments on order statistics arising from independent and nonidentically distributed random variables. For stochastic comparisons based on single-outlier and multiple-outlier models, we refer the reader to recent works by Khaledi and Kochar (2001), Hu et al. (2006), Wen et al. (2007), Xu et al. (2007), Hu et al. (2008), and the references therein.

In reliability engineering, an $n$-component system that works if and only if at least $k$ of the $n$ components work is called a $k$-out-of- $n$ system. Both parallel and series systems are special cases of the $k$-out-of- $n$ system. Let $X_{1: n} \leq X_{2: n} \leq \cdots \leq X_{n: n}$ denote the order statistics of the random variables $X_{1}, X_{2}, \ldots, X_{n}$. The lifetime of a $k$-out-of- $n$ system can be represented as $X_{n-k+1: n}$. It is of great interest to investigate the effect of the change in the parameters of the distributions of the parent observations on the system lifetime. In the literature, most of the work has focused on the effect of heterogeneity on the magnitude and dispersion of order statistics (cf. Bapat and Beg (1989), Kochar and Xu (2007), and Mao and Hu (2010)). However, not much work has been done on comparing the skewness of order statistics. The skewness of order statistics is critical in understanding the tail behavior of distributions, which is of interest in actuarial science and extreme value theory. For example, actuaries are always interested in the tail risks. Two well-known measures used in this area are value-at-risk (VaR) and expected shortfall (ES), which are closely related to the skewness of distributions. The more recent work of Kochar and $\mathrm{Xu}$ (2009) showed that the largest order statistic from a heterogeneous exponential sample is more skewed than that from a homogeneous exponential sample in the sense of the convex transform order. However, such a study has not been carried out for other order statistics.

In this paper we will compare order statistics according to the star ordering (denoted by ' $\geq_{\star}$ '). We show that, for the multiple-outlier exponential model, more heterogeneity among the scale parameters leads to more skewed order statistics. More specifically, let $X_{1}, \ldots, X_{p}$ be independent exponential random variables with a common hazard rate $\lambda_{1}$, and let $X_{p+1}, \ldots, X_{n}$ be another set of i.i.d. exponential random variables with hazard rate $\lambda_{2}$. Similarly, assume that $Y_{1}, \ldots, Y_{p}$ are independent exponential random variables with common hazard rate $\gamma_{1}$, and that $Y_{p+1}, \ldots, Y_{n}$ are independent exponential random variables with common hazard rate $\gamma_{2}$. We prove that

$$
\frac{\lambda_{(2)}}{\lambda_{(1)}} \geq \frac{\gamma_{(2)}}{\gamma_{(1)}} \quad \Longrightarrow \quad X_{k: n} \geq_{\star} Y_{k: n}
$$

for $1 \leq k \leq n$, where $\lambda_{(1)}=\min \left\{\lambda_{1}, \lambda_{2}\right\}$ and $\lambda_{(2)}=\max \left\{\lambda_{1}, \lambda_{2}\right\}$.

In particular, if $\gamma_{1}=\gamma_{2}$, in which case $Y_{1}, \ldots, Y_{n}$ constitutes a random sample from an exponential distribution, we prove that $X_{k: n} \geq_{\star} Y_{k: n}$. Furthermore, as applications, we provide sufficient and necessary conditions for comparing order statistics according to the hazard rate ordering and right-spread ordering in the proportional hazard rate model. That is, if $X_{1}, \ldots, X_{p}$ are independent random variables, each with survival function $\bar{F}^{\lambda_{1}}(x), X_{p+1}, \ldots, X_{n}$ is another set of independent random variables with common survival function $\bar{F}^{\lambda_{2}}(x)$, and $Y_{1}, \ldots, Y_{n}$ is a random sample from a distribution with survival function $\bar{F}^{\lambda}(x)$, then, if $F$ is DFR (decreasing failure rate), we prove that, for any $k \in\{1, \ldots, n\}$,

$$
X_{k: n} \geq{ }_{\mathrm{hr}} Y_{k: n}
$$


if

$$
\lambda \geq \hat{\lambda}=\left(\left(\begin{array}{l}
n \\
k
\end{array}\right)^{-1} \sum_{1 \leq i_{1}<\cdots<i_{k} \leq n} \lambda_{i_{1}} \cdots \lambda_{i_{k}}\right)^{1 / k}=\left(\left(\begin{array}{l}
n \\
k
\end{array}\right)^{-1} \sum_{l \in \mathbb{L}}\left(\begin{array}{c}
p \\
l
\end{array}\right)\left(\begin{array}{c}
n-p \\
k-l
\end{array}\right) \lambda_{1}^{l} \lambda_{2}^{k-l}\right)^{1 / k}
$$

where $\mathbb{L}=\{l: \max \{k-n+p, 0\} \leq l \leq \min \{p, k\}\}$ and ' $\geq$ hr' denotes the hazard rate order, and

$$
X_{k: n} \geq \mathrm{RS} Y_{k: n}
$$

if

$\lambda \geq \tilde{\lambda}$

$$
\begin{aligned}
& =\sum_{j=1}^{k} \frac{1}{n-j+1} / \sum_{j=n-k+1}^{n}(-1)^{j-n+k-1}\left(\begin{array}{l}
j-1 \\
n-k
\end{array}\right) \sum_{|S|=j} \frac{1}{\sum_{l \in S} \lambda_{l}} \\
& =\sum_{j=1}^{k} \frac{1}{n-j+1} / \sum_{j=n-k+1}^{n}(-1)^{j-n+k-1}\left(\begin{array}{l}
j-1 \\
n-k
\end{array}\right) \sum_{m \in \mathbb{M}}\left(\begin{array}{c}
p \\
m
\end{array}\right)\left(\begin{array}{l}
n-p \\
j-m
\end{array}\right) \frac{1}{m \lambda_{1}+(j-m) \lambda_{2}},
\end{aligned}
$$

where $S$ is a subset of $E=\{1,2, \ldots, n\},|S|$ denotes the cardinality of the set $S, \mathbb{M}=$ $\{m: \max \{j-n+p, 0\} \leq m \leq \min \{p, j\}\}$, and ' $\geq_{\mathrm{RS}}$ ' denotes the right-spread order.

Păltănea (2008) conjectured that condition (1.1) should imply the hazard rate order between order statistics from heterogeneous exponential samples and homogeneous exponential samples. Our results in this paper partially address this conjecture.

Throughout this paper, all random variables are assumed to be nonnegative and absolutely continuous. The inverse functions defined in this paper are assumed to be right continuous.

\section{Preliminaries}

In this section we recall some stochastic orders and majorization orders. Here and henceforth, the terms 'increasing' and 'decreasing' mean 'nondecreasing' and 'nonincreasing', respectively. All expectations and integrals are implicitly assumed to exist whenever they are written.

Assume that the random variables $X$ and $Y$ have distribution functions $F$ and $G$, survival functions $\bar{F}=1-F$ and $\bar{G}=1-G$, density functions $f$ and $g$, and failure rate functions $r_{X}=f / \bar{F}$ and $r_{Y}=g / \bar{G}$, respectively.

Definition 2.1. The random variable $X$ is said to be smaller than $Y$ in the convex transform order, denoted by $X \leq_{\mathrm{c}} Y$, if and only if $G^{-1} F(x)$ is convex in $x$ on the support of $X$.

If $X \leq_{\mathrm{c}} Y$ then $Y$ is more skewed than $X$, as explained in van Zwet (1970) and Marshall and Olkin (2007, p. 70). The convex transform order is also called the more IFR (increasing failure rate) order in reliability theory, since, when $f$ and $g$ exist, the convexity of $G^{-1} F(x)$ means that

$$
\frac{f\left(F^{-1}(u)\right)}{g\left(G^{-1}(u)\right)}=\frac{r_{X}\left(F^{-1}(u)\right)}{r_{Y}\left(G^{-1}(u)\right)}
$$

is increasing in $u \in[0,1]$. Thus, $X \leq_{\mathrm{c}} Y$ can be interpreted to mean that $X$ ages faster than $Y$ in some sense.

Definition 2.2. The random variable $X$ is said to be smaller than $Y$ in the star order, denoted by $X \leq_{\star} Y$ (or $F \leq_{\star} G$ ), if the function $G^{-1} F(x)$ is star shaped in the sense that $G^{-1} F(x) / x$ is increasing in $x$ on the support of $X$. 
The star order is also called the more IFRA (increasing failure rate in average) order in reliability theory, since the average failure of $F$ at $x$ is

$$
\bar{r}_{X}(x)=\frac{1}{x} \int_{0}^{x} r_{X}(u) \mathrm{d} u=\frac{-\ln \bar{F}(x)}{x} .
$$

Thus, $F \leq_{\star} G$ can be interpreted in terms of average failure rates, i.e.

$$
\frac{\bar{r}_{X}\left(F^{-1}(u)\right)}{\bar{r}_{Y}\left(G^{-1}(u)\right)}
$$

is increasing in $u \in(0,1]$. Note that $X$ has an increasing failure rate if and only if $F$ is star ordered with respect to the exponential distribution.

The function

$$
L_{X}(u)=\frac{1}{\mathrm{E}(X)} \int_{0}^{F^{-1}(u)} x \mathrm{~d} F(x)
$$

is known as the Lorenz curve in the economics literature. It is often used to express the inequality in incomes. Based on the Lorenz curve, the Lorenz order has been proposed in economics to compare income inequalities.

Definition 2.3. The random variable $X$ is said to be smaller than $Y$ in the Lorenz order, denoted by $X \leq$ Lorenz $Y$, if

$$
\frac{1}{\mathrm{E}(X)} \int_{0}^{F^{-1}(u)} x \mathrm{~d} F(x) \geq \frac{1}{\mathrm{E}(Y)} \int_{0}^{G^{-1}(u)} x \mathrm{~d} G(x) \quad \text { for all } u \in(0,1] .
$$

It is known in the literature (see Marshall and Olkin (2007, p. 69)) that

$$
X \leq_{\mathrm{c}} Y \quad \Longrightarrow \quad X \leq_{\star} Y \quad \Longrightarrow \quad X \leq_{\text {Lorenz }} Y \quad \Longrightarrow \quad \operatorname{cv}(X) \leq \operatorname{cv}(Y),
$$

where $\operatorname{cv}(X)=\sqrt{\operatorname{var}(x)} / \mathrm{E}(X)$ denotes the coefficient of variation of $X$.

All the above partial orders are scale invariant. Good discussions of the star order and Lorenz order can be found in Barlow and Proschan (1981, p. 105), Marshall and Olkin (2007, p. 73), and Kochar (1989).

A basic partial order used to compare variabilities in two distributions is the dispersive order defined as follows.

Definition 2.4. The random variable $X$ is said to be less dispersed than $Y$, denoted by $X \leq_{\text {disp }}$ $Y$, if

$$
F^{-1}(\beta)-F^{-1}(\alpha) \leq G^{-1}(\beta)-G^{-1}(\alpha)
$$

for all $0<\alpha \leq \beta<1$.

A weaker order called the right-spread order in Fernández-Ponce et al. (1998), or the excess wealth order in Shaked and Shanthikumar (1998), has also been proposed to compare the variabilities of two distributions.

Definition 2.5. The random variable $X$ is said to be less right spread than $Y$, denoted by $X \leq \mathrm{RS} Y$, if

$$
\int_{F^{-1}(p)}^{\infty} \bar{F}(x) \mathrm{d} x \leq \int_{G^{-1}(p)}^{\infty} \bar{G}(x) \mathrm{d} x \quad \text { for all } 0 \leq p \leq 1 .
$$


For more discussion on the dispersive order and the right-spread order, we refer the reader to Shaked and Shanthikumar (2007) and the references therein.

The hazard rate order and the usual stochastic order are also discussed in the sequel.

Definition 2.6. The random variable $X$ is said to be smaller than $Y$ in the hazard rate order, denoted by $X \leq{ }_{\mathrm{hr}} Y$, if $\bar{G}(x) / \bar{F}(x)$ is increasing in $x$.

Definition 2.7. The random variable $X$ is said to be smaller than $Y$ in the usual stochastic order, denoted by $X \leq$ st $Y$, if $\bar{F}(x) \leq \bar{G}(x)$ for all $x$.

We will also be using the concept of majorization in our discussion. Let $x_{(1)} \leq x_{(2)} \leq \cdots \leq$ $x_{(n)}$ be the increasing arrangement of the components of the vector $\boldsymbol{x}=\left(x_{1}, x_{2}, \ldots, x_{n}\right)$.

Definition 2.8. For vectors $\boldsymbol{x}, \boldsymbol{y} \in \mathbb{R}^{n}, \boldsymbol{x}$ is said to be

- majorized by $\boldsymbol{y}$, denoted by $\boldsymbol{x} \preceq_{\mathrm{m}} \boldsymbol{y}$, if

$$
\sum_{i=1}^{j} x_{(i)} \geq \sum_{i=1}^{j} y_{(i)}
$$

for $j=1, \ldots, n-1$ and $\sum_{i=1}^{n} x_{(i)}=\sum_{i=1}^{n} y_{(i)}$;

- weakly supermajorized by $\boldsymbol{y}$, denoted by $\boldsymbol{x} \preceq{ }^{\mathrm{w}} \boldsymbol{y}$, if

$$
\sum_{i=1}^{j} x_{(i)} \geq \sum_{i=1}^{j} y_{(i)}
$$

for $j=1, \ldots, n$;

- weakly submajorized by $\boldsymbol{y}$, denoted by $\boldsymbol{x} \preceq_{\mathrm{w}} \boldsymbol{y}$, if

$$
\sum_{i=j}^{n} x_{(i)} \leq \sum_{i=j}^{n} y_{(i)}
$$

for $j=1, \ldots, n$.

For extensive and comprehensive details on the theory of majorization orders and their applications, we refer the reader to the excellent book by Marshall and Olkin (1979).

The joint distribution functions of order statistics can be conveniently represented in terms of permanents when the underlying random variables are not identical. We refer the reader to Bapat and Kochar (1994), Hu et al. (2001), (2006), and Balakrishnan (2007) for related topics. For comprehensive details on the theory of permanents, we refer the reader to Minc (1978).

If $\boldsymbol{A}=\left(a_{i, j}\right)$ is an $n \times n$ matrix then the permanent of $\boldsymbol{A}$ is defined as

$$
\operatorname{perm}(\boldsymbol{A})=\sum_{\sigma} \prod_{i=1}^{n} a_{i, \sigma(i)},
$$

where the summation is taken over all permutations $\sigma=(\sigma(1), \ldots, \sigma(n))$ of $(1, \ldots, n)$. If $\boldsymbol{a}_{1}, \boldsymbol{a}_{2}, \ldots$ are column vectors then the permanent

$$
\operatorname{perm}(\boldsymbol{A})=[\underbrace{\boldsymbol{a}_{1}}_{r_{1}}, \underbrace{\boldsymbol{a}_{2}}_{r_{2}}, \ldots]
$$

is obtained by taking $r_{1}$ copies of $\boldsymbol{a}_{1}, r_{2}$ copies of $\boldsymbol{a}_{2}$, and so on. 
As with determinants, we could use Laplace expansions for the permanents along any row or column. For example, if $\boldsymbol{A}(i, j)$ denotes the matrix $\boldsymbol{A}$ with row $i$ and column $j$ deleted, then

$$
\begin{array}{ll}
\operatorname{perm}(\boldsymbol{A})=\sum_{j=1}^{n} a_{i j} \operatorname{perm}(\boldsymbol{A}(i, j)) & \text { for } i=1, \ldots, n, \\
\operatorname{perm}(\boldsymbol{A})=\sum_{i=1}^{n} a_{i j} \operatorname{perm}(\boldsymbol{A}(i, j)) & \text { for } j=1, \ldots, n .
\end{array}
$$

For mutually independent random variables $X_{1}, \ldots, X_{n}$, let $F_{i}$ and $\bar{F}_{i}$ be the distribution and survival functions of $X_{i}, i=1, \ldots, n$, respectively. The column vector $\left(F_{1}(x), \ldots, F_{n}(x)\right)^{\top}$ will be denoted by $\boldsymbol{F}(x)$. We define $\overline{\boldsymbol{F}}(x)$ similarly.

Note that the distribution function of an $(n-k+1)$-out-of- $n$ system has the following permanent expression (cf. Bapat and Beg (1989)). For $1 \leq k \leq n$ and $x \geq 0$,

$$
\begin{aligned}
F_{k, n}(x) & =\mathrm{P}\left(X_{k: n} \leq x\right) \\
& =\sum_{i=k}^{n} \mathrm{P}(\text { exactly } i X \text { s are less than or equal to } x) \\
& =\sum_{i=k}^{n} \frac{1}{i !(n-i) !}[\underbrace{\boldsymbol{F}(x)}_{i}, \underbrace{\overline{\boldsymbol{F}}(x)}_{n-i}] .
\end{aligned}
$$

Let $X_{1}, \ldots, X_{p}$ be independent exponential random variables with hazard rate $\lambda_{1}$, and let $X_{p+1}, \ldots, X_{n}$ be another set of i.i.d. exponential random variables with hazard rate $\lambda_{2}$. Then, for $1 \leq k \leq n$, the distribution function of $X_{k: n}$ for $x \geq 0$ is

$$
F_{k, n, \lambda}(x)=\sum_{i=k}^{n} \frac{1}{i !(n-i) !}[\underbrace{\boldsymbol{F}_{\lambda}(x)}_{i}, \underbrace{\overline{\boldsymbol{F}}_{\lambda}(x)}_{n-i}]_{p, n-p},
$$

where the subscript $p, n-p$ means that $p$ rows have hazard rate $\lambda_{1}$ and $n-p$ rows have hazard rate $\lambda_{2}$, and $\lambda=\left(\lambda_{1}, \lambda_{2}\right)$.

The density function of $X_{k: n}$ can be represented as

$$
f_{k, n, \lambda}(x)=\frac{1}{(k-1) !(n-k) !}[\underbrace{\boldsymbol{F}_{\lambda}(x)}_{k-1}, \boldsymbol{f}_{\lambda}(x), \underbrace{\overline{\boldsymbol{F}}_{\lambda}(x)}_{n-k}]_{p, n-p} .
$$

\section{Main result}

The following lemma, which is originally due to Saunders and Moran (1978, p. 429), will be used to derive the main result.

Lemma 3.1. Let $\left\{F_{\lambda} \mid \lambda \in \mathbb{R}\right\}$ be a class of distribution functions, such that $F_{\lambda}$ is supported on some interval $(a, b) \subseteq(0, \infty)$ and has density $f_{\lambda}$ which does not vanish on any subinterval of $(a, b)$. Then,

$$
F_{\lambda} \leq_{\star} F_{\lambda^{*}}, \quad \lambda \leq \lambda^{*},
$$

if and only if

$$
\frac{F_{\lambda}^{\prime}(x)}{x f_{\lambda}(x)} \text { is decreasing in } x,
$$

where $F_{\lambda}^{\prime}$ is the derivative of $F_{\lambda}$ with respect to $\lambda$. 
Now we are ready to present our main result, which is based on the well-known concepts of $\mathrm{TP}_{2}$ (totally positive of order 2 ) and $\mathrm{RR}_{2}$ (reversed total positivity of order 2 ). For two subsets $\Theta$ and $\Gamma$ of the real line, a real-valued function $h$ defined on $\Theta \times \Gamma$ is said to be $\mathrm{TP}_{2}$ or $\mathrm{RR}_{2}$ if $h(x, y) h\left(x^{\prime}, y^{\prime}\right) \geq h\left(x, y^{\prime}\right) h\left(x^{\prime}, y\right)$ or $h(x, y) h\left(x^{\prime}, y^{\prime}\right) \leq h\left(x, y^{\prime}\right) h\left(x^{\prime}, y\right)$, respectively, whenever $x \leq x^{\prime}, y \leq y^{\prime}, x, x^{\prime} \in \Theta$, and $y, y^{\prime} \in \Gamma$. For more discussion on total positivity, we refer the reader to the excellent book by Karlin (1968).

Theorem 3.1. Let $X_{1}, \ldots, X_{p}$ be i.i.d. exponential random variables with hazard rate $\lambda_{1}$, and let $X_{p+1}, \ldots, X_{n}$ be another set of i.i.d. exponential random variables with hazard rate $\lambda_{2}$. Let $Y_{1}, \ldots, Y_{p}$ be i.i.d. exponential random variables with hazard rate $\gamma_{1}$, and let $Y_{p+1}, \ldots, Y_{n}$ be another set of i.i.d. exponential random variables with hazard rate $\gamma_{2}$. Then, for $k=1, \ldots, n$,

$$
\frac{\lambda_{(2)}}{\lambda_{(1)}} \geq \frac{\gamma_{(2)}}{\gamma_{(1)}} \quad \Longrightarrow \quad X_{k: n} \geq_{\star} Y_{k: n} .
$$

Proof. Case 1: $\lambda_{1}+\lambda_{2}=\gamma_{1}+\gamma_{2}$. Without loss of generality, let

$$
\lambda_{1}+\lambda_{2}=\gamma_{1}+\gamma_{2}=1 \text {. }
$$

Note that the assumption in this case implies that

$$
\left(\lambda_{1}, \lambda_{2}\right) \succeq_{\mathrm{m}}\left(\gamma_{1}, \gamma_{2}\right) \text {. }
$$

Now, let $\lambda_{1}=\lambda \geq \lambda_{2}$ and $\gamma_{1}=\gamma \geq \gamma_{2}$. According to Lemma 3.1, it is enough to show that

$$
\frac{F_{k, n, \lambda}^{\prime}(x)}{x f_{k, n, \lambda}(x)}
$$

is decreasing in $x$ for $\lambda \in\left[\frac{1}{2}, 1\right)$.

Note that

$$
\begin{aligned}
F_{k, n, \lambda}^{\prime}= & \sum_{i=k}^{n} \frac{i}{i !(n-i) !}[\underbrace{\boldsymbol{F}_{\lambda}(x)}_{i-1}, F_{\lambda}^{\prime}(x), \underbrace{\overline{\boldsymbol{F}}_{\lambda}(x)}_{n-i}]_{p, n-p} \\
& -\sum_{i=k}^{n} \frac{n-i}{i !(n-i) !}[\underbrace{\boldsymbol{F}_{\lambda}(x)}_{i}, F_{\lambda}^{\prime}(x), \underbrace{\overline{\boldsymbol{F}}_{\lambda}(x)}_{n-i-1}]_{p, n-p} \\
= & \sum_{i=k}^{n} \frac{1}{(i-1) !(n-i) !}[\underbrace{\boldsymbol{F}_{\lambda}(x)}_{i-1}, F_{\lambda}^{\prime}(x), \underbrace{\overline{\boldsymbol{F}}_{\lambda}(x)}_{n-i}]_{p, n-p} \\
& -\sum_{i=k}^{n-1} \frac{1}{i !(n-i-1) !}[\underbrace{\boldsymbol{F}_{\lambda}(x)}_{i}, F_{\lambda}^{\prime}(x), \underbrace{\overline{\boldsymbol{F}}_{\lambda}(x)}_{n-i-1}]_{p, n-p} \\
= & \sum_{i=k}^{n} \frac{1}{(i-1) !(n-i) !}[\underbrace{\boldsymbol{F}_{\lambda}(x)}_{i-1}, F_{\lambda}^{F_{\lambda}^{\prime}}(x), \underbrace{\overline{\boldsymbol{F}}_{\lambda}(x)}_{n-i}]_{p, n-p} \\
& -\sum_{i=k+1}^{n} \frac{1}{(i-1) !(n-i) !}[\underbrace{\boldsymbol{F}_{\lambda}(x)}_{i-1}, F_{\lambda}^{\prime}(x), \underbrace{\overline{\boldsymbol{F}}_{\lambda}(x)}_{n-i}]_{p, n-p} \\
= & \frac{1}{(k-1) !(n-k) !}[\underbrace{\boldsymbol{F}_{\lambda}(x)}_{k-1}, F_{\lambda}^{\prime}(x), \underbrace{\overline{\boldsymbol{F}}_{\lambda}(x)}_{n-k}]_{p, n-p} .
\end{aligned}
$$


Using the Laplace expansion, it follows that

$$
\begin{aligned}
\frac{F_{k, n, \lambda}^{\prime}(x)}{x f_{k, n, \lambda}(x)}= & \left(p \mathrm{e}^{-\lambda x}[\underbrace{\boldsymbol{F}_{\lambda}(x)}_{k-1}, \underbrace{\overline{\boldsymbol{F}}_{\lambda}(x)}_{n-k}]_{p-1, n-p}-(n-p) \mathrm{e}^{-(1-\lambda) x}[\underbrace{\boldsymbol{F}_{\lambda}(x)}_{k-1}, \underbrace{\overline{\boldsymbol{F}}_{\lambda}(x)}_{n-k}]_{p, n-p-1}\right) \\
\times & \left(p \lambda \mathrm{e}^{-\lambda x}[\underbrace{\boldsymbol{F}_{\lambda}(x)}_{k-1}, \underbrace{\overline{\boldsymbol{F}}_{\lambda}(x)}_{n-k}]_{p-1, n-p}\right. \\
& \left.+(1-\lambda)(n-p) \mathrm{e}^{-(1-\lambda) x}[\underbrace{\boldsymbol{F}_{\lambda}(x)}_{k-1}, \underbrace{\overline{\boldsymbol{F}}_{\lambda}(x)}_{n-k}]_{p, n-p-1}\right)^{-1} \\
= & \triangle(x, \lambda)\left(\lambda \Delta(x, \lambda)+(n-p) \mathrm{e}^{-(1-\lambda) x}[\underbrace{\boldsymbol{F}_{\lambda}(x)}_{k-1}, \underbrace{\overline{\boldsymbol{F}}_{\lambda}(x)}_{n-k}]_{p, n-p-1}\right)^{-1},
\end{aligned}
$$

where

$$
\triangle(x, \lambda)=p \mathrm{e}^{-\lambda x}[\underbrace{\boldsymbol{F}_{\lambda}(x)}_{k-1}, \underbrace{\overline{\boldsymbol{F}}_{\lambda}(x)}_{n-k}]_{p-1, n-p}-(n-p) \mathrm{e}^{-(1-\lambda) x}[\underbrace{\boldsymbol{F}_{\lambda}(x)}_{k-1}, \underbrace{\overline{\boldsymbol{F}}_{\lambda}(x)}_{n-k}]_{p, n-p-1} .
$$

Hence, it is enough to prove that

$$
h(x)=\mathrm{e}^{-\lambda x}[\underbrace{\boldsymbol{F}_{\lambda}(x)}_{k-1}, \underbrace{\overline{\boldsymbol{F}}_{\lambda}(x)}_{n-k}]_{p-1, n-p} / \mathrm{e}^{-(1-\lambda) x}[\underbrace{\boldsymbol{F}_{\lambda}(x)}_{k-1}, \underbrace{\overline{\boldsymbol{F}}_{\lambda}(x)}_{n-k}]_{p, n-p-1}
$$

is decreasing in $x \geq 0$ for $\frac{1}{2} \leq \lambda<1$.

By Laplace expansion along the first $k-1$ columns of the permanent, it holds that

$$
\begin{aligned}
h(x)= & \left(\mathrm{e}^{(1-\lambda) x} \sum_{i \in \mathbb{I}}\left(\begin{array}{c}
n-p \\
i
\end{array}\right)\left(\begin{array}{c}
p-1 \\
k-1-i
\end{array}\right)[\underbrace{\boldsymbol{F}_{\lambda}(x)}_{k-1}]_{k-1-i, i}[\underbrace{\overline{\boldsymbol{F}}_{\lambda}(x)}_{n-k}]_{p-k+i, n-p-i}\right) \\
& \times\left(\mathrm{e}^{\lambda x} \sum_{j \in \mathbb{J}}\left(\begin{array}{c}
n-p-1 \\
j
\end{array}\right)\left(\begin{array}{c}
p \\
k-1-j
\end{array}\right)[\underbrace{\boldsymbol{F}_{\lambda}(x)}_{k-1}]_{k-1-j, j}[\underbrace{\overline{\boldsymbol{F}}_{\lambda}(x)}_{n-k}]_{p-k+j+1, n-p-j-1}\right)^{-1} \\
= & \left(\mathrm{e}^{(1-\lambda) x} \sum_{i \in \mathbb{I}}\left(\begin{array}{c}
n-p \\
i
\end{array}\right)\left(\begin{array}{c}
p-1 \\
k-1-i
\end{array}\right)\left(1-\mathrm{e}^{-\lambda x}\right)^{k-1-i}\left(1-\mathrm{e}^{-(1-\lambda) x}\right)^{i} \mathrm{e}^{-\lambda x(p-k+i)}\right. \\
& \times\left(\mathrm{e}^{\lambda x} \sum_{j \in \mathbb{J}}\left(\begin{array}{c}
n-(1-\lambda)(n-p-i) x \\
j-1
\end{array}\right)\left(\begin{array}{c}
p \\
k-1-j
\end{array}\right)\left(1-\mathrm{e}^{-\lambda x}\right)^{k-1-j}\left(1-\mathrm{e}^{-(1-\lambda) x}\right)^{j}\right. \\
\times & \left(\sum_{i \in \mathbb{I}}\left(\begin{array}{c}
n-p \\
i
\end{array}\right)\left(\begin{array}{c}
p-1 \\
k-1-i
\end{array}\right) \delta^{i}(x, \lambda)\right)\left(\sum_{j \in \mathbb{J}}\left(\begin{array}{c}
n-p-1 \\
j
\end{array}\right)\left(\begin{array}{c}
p \\
k-1-j
\end{array}\right) \delta^{j}(x, \lambda)\right)^{-(1-\lambda)(n-p-1-j) x}
\end{aligned}
$$

where

$$
\begin{gathered}
\delta(x, \lambda)=\frac{\mathrm{e}^{(1-\lambda) x}-1}{\mathrm{e}^{\lambda x}-1}, \\
\mathbb{I}=\{i: \max \{k-p, 0\} \leq i \leq \min \{n-p, k-1\}\},
\end{gathered}
$$


and

$$
\mathbb{J}=\{j: \max \{k-p-1,0\} \leq j \leq \min \{n-p-1, k-1\}\} .
$$

Now, it is enough to prove that

$$
r(x, l)=\sum_{i \in \mathbb{I}}\left(\begin{array}{c}
n-p-l \\
i
\end{array}\right)\left(\begin{array}{l}
p-1+l \\
k-1-i
\end{array}\right) \delta^{i}(x, \lambda)
$$

is $\mathrm{TP}_{2}$ in $\{x, l\} \in \mathbb{R}_{+} \times\{0,1\}$. It is easy to see that $\delta(x, \lambda)$ is decreasing in $x \in \mathbb{R}_{+}$for $\frac{1}{2} \leq \lambda<1$, and, hence, $\delta^{i}(x, \lambda)$ is $\mathrm{RR}_{2}$ in $(x, i) \in \mathbb{R}_{+} \times \mathbb{I}$. Since

$$
\left(\begin{array}{c}
n-p-l \\
i
\end{array}\right)\left(\begin{array}{l}
p-1+l \\
k-1-i
\end{array}\right)
$$

is $\mathrm{RR}_{2}$ in $i \times l \in \mathbb{I} \times\{0,1\}$, using the basic composition formula (see $\operatorname{Karlin}(1968, \mathrm{p}$. 17)), the required result follows.

Case 2: $\lambda_{1}+\lambda_{2} \neq \gamma_{1}+\gamma_{2}$. Without loss of generality, assume that

$$
\lambda_{1}+\lambda_{2}=c\left(\gamma_{1}+\gamma_{2}\right)
$$

where $c>0$ is a scalar. Now

$$
\left(\lambda_{1}, \lambda_{2}\right) \succeq_{\mathrm{m}}\left(c \gamma_{1}, c \gamma_{2}\right) \text {. }
$$

Now assume that $Z_{k: n}$ is the $k$ th order statistic of the exponential random variables $Z_{1}, \ldots, Z_{p}$ with hazard rate $c \gamma_{1}$ and $Z_{p+1}, \ldots, Z_{n}$ with hazard rate $c \gamma_{2}$. It follows from case 1 that

$$
X_{k: n} \geq_{\star} Z_{k: n} .
$$

Since the star order is scale invariant, it follows that

$$
X_{k: n} \geq_{\star} Y_{k: n} .
$$

Remark. Note that the condition given in Theorem 3.1 is quite general. For example, it is weaker than any of the following conditions:

(a) $\left(\lambda_{1}, \lambda_{2}\right) \succeq_{\mathrm{m}}\left(\gamma_{1}, \gamma_{2}\right)$;

(b) $\left(\log \left(\lambda_{1}\right), \log \left(\lambda_{2}\right)\right) \succeq_{\mathrm{m}}\left(\log \left(\gamma_{1}\right), \log \left(\gamma_{2}\right)\right)$;

(c) $\left(1 / \lambda_{1}, 1 / \lambda_{2}\right) \succeq_{\mathrm{m}}\left(1 / \gamma_{1}, 1 / \gamma_{2}\right)$.

The reader may wonder whether Theorem 3.1 could be extended to the general case when the $n$ parameters are all different and the sets of parameters majorize each order. The following result serves as a counterexample.

Example 3.1. Let $\left(X_{1}, X_{2}, X_{3}\right)$ be an independent exponential random vector with parameters $(1,2,9)$, and let $\left(Y_{1}, Y_{2}, Y_{3}\right)$ be another independent exponential random vector with parameters $(1,5,6)$. It is easy to see that

$$
(1,2,9) \succeq_{\mathrm{m}}(1,5,6) \text {. }
$$

Using MATHEMATICA ${ }^{\circledR}$ to compute the coefficients of variation of $X_{3: 3}$ and $Y_{3: 3}$, we have

$$
\operatorname{cv}\left(X_{3: 3}\right)=0.815396 \text { and } \operatorname{cv}\left(Y_{3: 3}\right)=0.921265,
$$


which means that

$$
\operatorname{cv}\left(X_{3: 3}\right) \leq \operatorname{cv}\left(Y_{3: 3}\right)
$$

Hence,

$$
X_{3: 3} \Varangle_{\star} Y_{3: 3} \text {. }
$$

As a direct consequence of Theorem 3.1, we see that the order statistics from multiple-outlier exponential models are more skewed than the corresponding statistics from the homogeneous exponential model in the sense of the star ordering.

Corollary 3.1. Let $X_{1}, \ldots, X_{p}$ be independent exponential random variables with hazard rate $\lambda_{1}$, and let $X_{p+1}, \ldots, X_{n}$ be another set of independent exponential random variables with common hazard rate $\lambda_{2}$. Let $Y_{1}, \ldots, Y_{n}$ be a random sample from any exponential distribution. Then, for $1 \leq k \leq n$,

$$
X_{k: n} \geq_{*} Y_{k: n} .
$$

Since the star order implies the Lorenz order, the following result is of interest in economics.

Corollary 3.2. Let $X_{1}, \ldots, X_{p}$ be independent exponential random variables with common hazard rate $\lambda_{1}$, and let $X_{p+1}, \ldots, X_{n}$ be another set of independent exponential random variables with common hazard rate $\lambda_{2}$. Let $Y_{1}, \ldots, Y_{n}$ be a random sample from any exponential distribution. Then,

$$
X_{k: n} \geq_{\text {Lorenz }} Y_{k: n}
$$

\section{Applications in proportional hazard rate models}

Let $X_{1}, X_{2}, \ldots, X_{n}$ be independent random variables. If the survival function of $X_{i}$ can be expressed as

$$
\bar{F}_{i}(x)=[\bar{F}(x)]^{\lambda_{i}}, \quad \lambda_{i}>0,
$$

for $i=1, \ldots, n$, where $\bar{F}(x)$ is the survival function of the baseline random variable, then the $X_{i}$ are said to follow the proportional hazard rate (PHR) model.

If $r(x)$ denotes the hazard rate corresponding to the baseline distribution $F$ then the hazard rate of $X_{i}$ is

$$
r_{i}(x)=\lambda_{i} r(x), \quad i=1,2, \ldots, n .
$$

Another equivalent expression for (4.1) is

$$
\bar{F}_{i}(x)=\mathrm{e}^{-\lambda_{i} R(x)}, \quad i=1,2, \ldots, n,
$$

where $R(x)=\int_{0}^{x} r(t) \mathrm{d} t$ is the cumulative hazard rate of $X$. Many well-known models are special cases of the PHR model. In the following, we will discuss some applications of our main theorem to PHR models.

Lemma 4.1. Let $X_{1}, \ldots, X_{p}$ be independent exponential random variables with common hazard rate $\lambda_{1}$, and let $X_{p+1}, \ldots, X_{n}$ be another set of i.i.d. exponential random variables with hazard rate $\lambda_{2}$. Let $Y_{1}, \ldots, Y_{n}$ be independent exponential random variables with common hazard rate $\hat{\lambda}$, satisfying

$$
\hat{\lambda} \geq\left[\left(\begin{array}{l}
n \\
k
\end{array}\right)^{-1} \sum_{l \in \mathbb{L}}\left(\begin{array}{l}
p \\
l
\end{array}\right)\left(\begin{array}{c}
n-p \\
k-l
\end{array}\right) \lambda_{1}^{l} \lambda_{2}^{k-l}\right]^{1 / k},
$$


where

$$
\mathbb{L}=\{l: \max \{k-n+p, 0\} \leq l \leq \min \{p, k\}\} .
$$

Then, the following equivalent results hold:

(a) $X_{k: n} \geq \operatorname{disp} Y_{k: n}$;

(b) $X_{k: n} \geq \mathrm{hr} Y_{k: n}$;

(c) $X_{k: n} \geq_{s t} Y_{k: n}$.

Proof. The proof follows from Theorem 3 of Ahmed et al. (1986), which states that, under the condition that $X_{k: n} \geq_{*} Y_{k: n}$, a result proved in our Corollary 3.1,

$$
X_{k: n} \geq_{\mathrm{st}} Y_{k: n} \quad \Longrightarrow \quad X_{k: n} \geq_{\text {disp }} Y_{k: n}
$$

Bon and Păltănea (2006) showed that

$$
X_{k: n} \geq_{\text {st }} Y_{k: n}
$$

is equivalent to

$$
\hat{\lambda} \geq\left(\left(\begin{array}{l}
n \\
k
\end{array}\right)^{-1} \sum_{1 \leq i_{1}<\cdots<i_{k} \leq n} \lambda_{i_{1}} \cdots \lambda_{i_{k}}\right)^{1 / k}=\left(\left(\begin{array}{l}
n \\
k
\end{array}\right)^{-1} \sum_{l \in \mathbb{L}}\left(\begin{array}{l}
p \\
l
\end{array}\right)\left(\begin{array}{c}
n-p \\
k-l
\end{array}\right) \lambda_{1}^{l} \lambda_{2}^{k-l}\right)^{1 / k}
$$

where $\mathbb{L}=\{l: \max \{k-n+p, 0\} \leq l \leq \min \{p, k\}\}$.

Combining the above statements, completes the proof of (a).

Observing that $Y_{k: n}$ has increasing failure rate (see Barlow and Proschan (1981, p. 108)), it follows from Bagai and Kochar (1986) (see also Theorem 3.B.20 of Shaked and Shanthikumar (2007)) that (a) implies (b). The result that (b) implies (c) is obvious.

Observing that $X_{k: n} \geq_{\text {disp }} Y_{k: n}$ implies that $X_{k: n} \geq_{s t} Y_{k: n}$ in the case of distributions with a common left-hand point of support proves the equivalence of (a) and (c) from Corollary 3.1. Hence, the required result follows.

Using arguments similar to those in the proof of Theorem 2.3 of Kochar and Xu (2007), we can derive the following result from Lemma 4.1.

Theorem 4.1. Let $X_{1}, \ldots, X_{p}$ be independent random variables with common survival function $\bar{F}^{\lambda_{1}}(x)$, and let $X_{p+1}, \ldots, X_{n}$ be another set of independent random variables with common survival function $\bar{F}^{\lambda_{2}}(x)$. Let $Y_{1}, \ldots, Y_{n}$ be a random sample from a distribution with survival function $\bar{F}^{\hat{\lambda}}(x)$. If $F$ has a DFR distribution then, for $1 \leq k \leq n$,

$$
X_{k: n} \geq_{\text {disp }} Y_{k: n}
$$

Next, we will derive a parallel result for the right-spread order.

Lemma 4.2. Let $X_{1}, \ldots, X_{p}$ be independent exponential random variables with common hazard rate $\lambda_{1}$, and let $X_{p+1}, \ldots, X_{n}$ be another set of i.i.d. exponential random variables with hazard rate $\lambda_{2}$. Let $Y_{1}, \ldots, Y_{n}$ be independent exponential random variables with common 
hazard rate $\tilde{\lambda}$, satisfying the condition that

$$
\begin{aligned}
\tilde{\lambda} \geq & \sum_{j=1}^{k} \frac{1}{n-j+1} \\
& \times\left(\sum_{j=n-k+1}^{n}(-1)^{j-n+k-1}\left(\begin{array}{l}
j-1 \\
n-k
\end{array}\right) \sum_{m \in \mathbb{M}}\left(\begin{array}{c}
p \\
m
\end{array}\right)\left(\begin{array}{c}
n-p \\
j-m
\end{array}\right) \frac{1}{m \lambda_{1}+(j-m) \lambda_{2}}\right)^{-1},
\end{aligned}
$$

where

$$
\mathbb{M}=\{m: \max \{j-n+p, 0\} \leq m \leq \min \{p, j\}\} .
$$

Then, for $1 \leq k \leq n$, the following equivalent statements hold:

(a) $X_{k: n} \geq_{\mathrm{RS}} Y_{k: n}$;

(b) $\mathrm{E}\left(X_{k: n}\right) \geq \mathrm{E}\left(Y_{k: n}\right)$.

Proof. The proof follows from Theorem 4.3 of Fernández-Ponce et al. (1998) and Corollary 3.1. That is, $X_{k: n} \geq_{\mathrm{RS}} Y_{k: n}$ holds if $\mathrm{E}\left(X_{k: n}\right) \geq \mathrm{E}\left(Y_{k: n}\right)$.

Note that (see Balakrishnan (2007, p. 54)),

$$
\mathrm{E}\left(X_{k: n}\right)=\sum_{j=n-k+1}^{n}(-1)^{j-n+k-1}\left(\begin{array}{l}
j-1 \\
n-k
\end{array}\right) \sum_{|S|=j} \frac{1}{\sum_{l \in S} \lambda_{l}},
$$

where $S$ is a subset of $E=\{1,2, \ldots, n\}$, and $|S|$ denotes the cardinality of the set $S$. Since

$$
\mathrm{E}\left(Y_{k: n}\right)=\frac{1}{\tilde{\lambda}} \sum_{j=1}^{k} \frac{1}{n-j+1}
$$

it holds that

$$
\mathrm{E}\left(X_{k: n}\right) \geq \mathrm{E}\left(Y_{k: n}\right)
$$

which is equivalent to

$$
\frac{1}{\tilde{\lambda}} \sum_{j=1}^{k} \frac{1}{n-j+1} \leq \sum_{j=n-k+1}^{n}(-1)^{j-n+k-1}\left(\begin{array}{l}
j-1 \\
n-k
\end{array}\right) \sum_{|S|=j} \frac{1}{\sum_{l \in S} \lambda_{l}},
$$

i.e.

$$
\begin{aligned}
\tilde{\lambda} \geq & \sum_{j=1}^{k} \frac{1}{n-j+1}\left(\sum_{j=n-k+1}^{n}(-1)^{j-n+k-1}\left(\begin{array}{l}
j-1 \\
n-k
\end{array}\right) \sum_{|S|=j} \frac{1}{\sum_{l \in S} \lambda_{l}}\right)^{-1} \\
= & \sum_{j=1}^{k} \frac{1}{n-j+1} \\
& \times\left(\sum_{j=n-k+1}^{n}(-1)^{j-n+k-1}\left(\begin{array}{c}
j-1 \\
n-k
\end{array}\right) \sum_{m \in \mathbb{M}}\left(\begin{array}{c}
p \\
m
\end{array}\right)\left(\begin{array}{c}
n-p \\
j-m
\end{array}\right) \frac{1}{m \lambda_{1}+(j-m) \lambda_{2}}\right)^{-1},
\end{aligned}
$$

where $\mathbb{M}=\{m: \max \{j-n+p, 0\} \leq m \leq \min \{p, j\}\}$. Hence, the result follows since right-spread ordering implies the ordering of the expectations of the random variables under the given conditions. 
Using arguments similar to those in Theorem 4.5 of Kochar and $\mathrm{Xu}$ (2009), we can extend the above result to the PHR models.

Theorem 4.2. Let $X_{1}, \ldots, X_{p}$ be independent random variables with common survival function $\bar{F}^{\lambda_{1}}(x)$, and let $X_{p+1}, \ldots, X_{n}$ be another set of independent random variables with common survival function $\bar{F}^{\lambda_{2}}(x)$. Let $Y_{1}, \ldots, Y_{n}$ be a random sample from a distribution with survival function $\bar{F}^{\tilde{\lambda}}(x)$. If $F$ is DFR then, for $1 \leq k \leq n$,

$$
X_{k: n} \geq \mathrm{RS} Y_{k: n} \text {. }
$$

\section{Acknowledgement}

The authors are grateful to an anonymous referee for constructive comments which led to an improved version of the paper.

\section{References}

Ahmed, A. N., Alzaid, A., Bartoszewicz, J. and Kochar, S. C. (1986). Dispersive and superadditive ordering. Adv. Appl. Prob. 18, 1019-1022.

Arnold, B. C. And Groeneveld, R. A. (1995). Measuring skewness with respect to the mode. Amer. Statistician 49, 34-38.

BagaI, I. AND Kochar, S. (1986). On tail-ordering and comparison of failures rates. Commun. Statist. Theory Meth. 15, 1377-1388.

Balakrishnan, N. (2007). Permanents, order statistics, outliers, and robustness. Revista Matemática Complutense 20, 7-107.

Balakrishnan, N. ANd RaO, C. R. (eds) (1998a). Handbook of Statistics 16. Elsevier, New York.

Balakrishnan, N. ANd RaO, C. R. (eds) (1998b). Handbook of Statistics 17. Elsevier, New York.

BAPAT, R. B. AND BEG, M. I. (1989). Order statistics for nonidentically distributed variables and permanents. Sankhya $A$ 51, 79-93.

Bapat, R. B. AND Kochar, S. C. (1994). On likelihood-ratio ordering of order statistics. Linear Algebra Appl. 199, 281-291.

Barlow, R. E. And Proschan, F. (1981). Statistical Theory of Reliability and Life Testing. Holt, Rinehart and Winston, New York.

Bon, J.-L. AND PǍLtǎnea, E. (2006). Comparison of order statistics in a random sequence to the same statistics with i.i.d. variables. ESAIM Prob. Statist. 10, 1-10.

David, H. A. And Nagaraja, H. N. (2003). Order Statistics, 3rd edn. Wiley Interscience, Hoboken, NJ.

Fernández-Ponce, J. M., Kochar, S. C. And Muñoz-Perez, J. (1998). Partial orderings of distributions based on right-spread functions. J. Appl. Prob. 35, 221-228.

Hu, T., Lu, Q. AND WEN, S. (2008). Some new results on likelihood ratio orderings for spacings of heterogeneous exponential random variables. Commun. Statist. Theory Meth. 37, 2506-2515.

Hu, T., WANG, F. AND ZHU, Z. (2006). Stochastic comparisons and dependence of spacings from two samples of exponential random variables. Commun. Statist. Theory Meth. 35, 979-988.

Hu, T., ZHU, Z. AND WEI, Y. (2001). Likelihood ratio and mean residual life orders for order statistics of heterogeneous random variables. Prob. Eng. Inf. Sci. 15, 259-272.

Karlin, S. (1968). Total Positivity, Vol. I. Stanford University Press.

Khaledi, B. E. ANd Kochar, S. C. (2001). Stochastic properties of spacings in a single-outlier exponential model. Prob. Eng. Inf. Sci. 15, 401-408.

Kochar, S. C. (1989). On extensions of DMRL and related partial orderings of life distributions. Commun. Statist. Stoch. Models 5, 235-245.

Kochar, S. AND XU, M. (2007). Stochastic comparisons of parallel systems when components have proportional hazard rates. Prob. Eng. Inf. Sci. 21, 597-609.

Kochar, S. AND XU, M. (2009). Comparisons of parallel systems according to the convex transform order. J. Appl. Prob. 46, 342-352.

Mao, T. And Hu, T. (2010). Equivalent characterizations on orderings of order statistics and sample ranges. Prob. Eng. Inf. Sci. 24, 245-262.

Marshall, A. W. And Olkin, I. (1979). Inequalities: Theory of Majorization and Its Applications. Academic Press, New York.

Marshall, A. W. and OlKin, I. (2007). Life Distributions. Springer, New York. 
Minc, H. (1978). Permanents (Encyclopaedia Math. Appl. 6). Addison-Wesley, Reading, MA.

OJA, H. (1981). On location, scale, skewness and kurtosis of univariate distributions. Scand. J. Statist. 8, 154-168.

PǍltǎnea, E. (2008). On the comparison in hazard rate ordering of fail-safe systems. J. Statist. Planning Inf. 138, 1993-1997.

Saunders, I. W. And Moran, P. A. P. (1978). On the quantiles of the gamma and F distributions. J. Appl. Prob. 15, 426-432.

Shaked, M. and Shantikumar, J. G. (1998). Two variability orders. Prob. Eng. Inf. Sci. 12, 1-23.

Shaked, M. And Shanthikumar, J. G. (2007). Stochastic Orders and Their Applications. Springer, New York.

VAn Zwet, W. R. (1970). Convex Transformations of Random Variables (Math. Centre Tracts 7), 2nd edn. Mathematical Centre, Amsterdam.

WEN, S., LU, Q. AND HU, T. (2007). Likelihood ratio orderings of spacings of heterogeneous exponential random variables. J. Multivariate Anal. 98, 743-756.

XU, M., LI, X., ZHAO, P. AND LI, Z. (2007). Likelihood ratio order of m-spacings in multiple-outlier models. Commun. Statist. Theory Meth. 36, 1507-1525. 